\title{
Measured radiation sensitivity of Silica-on-Silicon and Silicon-on-insulator micro-photonic devices for potential space application
}

\author{
Pieter Dumon $^{a}$, Roel Baets ${ }^{a}$, Roman Kappeler $^{b}$, Daniel Barros $^{b}$, \\ Iain McKenzie ${ }^{b}$ and Dominic Doyle ${ }^{b}$ \\ ${ }^{a}$ Photonics Research Group, Dept. of Information Technology, Ghent University - IMEC, \\ Sint-Pietersnieuwstraat 41, 9000 Gent, Belgium \\ ${ }^{b}$ European Space Agency - European Space Research and Technology Center, Noordwijk, The \\ Netherlands
}

\begin{abstract}
Silica on Silicon (SoS) and Silicon on insulator (SOI) fabrication technologies are now yielding efficient photonic devices that have potential uses (e.g. routing and switching) in multigigabit optical backplane interconnect applications. The ever increasing demands for higher speed data handling and greater throughput on board both operational and experimental satellites necessitate an examination of these technologies for their robustness and performance in a space environment. One of the main environmental stressors on electronic and photonic components is the incident radiation flux. This paper reports results from the experimental testing of two classes of photonic devices; namely a SoS arrayed waveguide grating (AWG), and a SOI ring resonator. For a total ionizing dose of $300 \mathrm{kRad}$ Co60 gamma irradiation, the SOI ring resonator showed induced spectral shifts as lower than $0.4 \mathrm{pm} / \mathrm{kRad}$, and the SoS AWG showed a maximum shift of $0.03 \mathrm{pm} / \mathrm{kRad}$ in one channel. The relatively low AWG radiation sensitivity tempt us to say that these devices could be considered radiation hard for the telecom CL wavelength band $(1550 \mathrm{~nm})$ in which these measurements were made.
\end{abstract}

Keywords: radiation hardness, photonic integrated circuit, Silicon-on-insulator, Space optics

\section{INTRODUCTION}

Photonic integrated circuits (PICs) already used in terrestial applications can in theory deliver functionality, performance and flexibility for on-board interconnect systems in satellites while meeting the weight, space and power constraints imposed by space applications. A multigigabit optical backplane interconnect can be built around integrated passive wavelength routing devices. ${ }^{1}$

Arrayed waveguide grating devices are commercially available today for telecommunication applications. ${ }^{2}$ These components are based on mature planar waveguiding technology in Silica-on-Silicon (SoS). Their performance is very well suited for interconnect applications too and their low weight and small space is an advantage. The only power needed by a non-reconfigurable device is that needed for thermal control. A backplane system with basic functionality can therefore be built in a very elegant way, using such devices. However, when one wants more complex interconnect communication schemes, such as a combination of unicasting and multicasting operation, the number of needed components grows due to the low integration level of current PICs, based on low index contrast systems. In order to address this limitation, high-index contrast technology is under active research, shrinking components by a few orders of magnitude. Using these technologies, a complex functionality can be integrated on one small chip, increasing vibration tolerance and decreasing weight and needed space and power. Here we use Silicon-on-insulator (SOI) components. Waveguides in this technology have submicron dimensions and are fabricated with CMOS-based processes. ${ }^{3}$ CMOS technology has a distinct benefit for use in space due to its reduced latch up sensitivity. ${ }^{4}$

e-mail: Pieter.Dumon@intec.UGent.be 
Radiation hardness of these components is a major concern with respect to space operation. Radiationmatter interaction gives rise to refractive index and absorption changes. Non-optimal optical transmission may result and basically the bit error rate in a communication system will rise. If these changes are considerable, components in an optical backplane application may therefore have to be actively tuned or recalibrated. Because refractive index changes have a larger impact in high-index contrast components, we expect SOI devices to be less radiation-hard than SoS components. We investigated the radiation-induced effects in a commercially available SoS arrayed waveguide grating device and several experimental SOI ring resonator devices. Our main interest was to study total ionizing dose effects on the optical performance of these PIC components. Also, relaxation in the first hours after radiation was monitored. Components were radiated by a $\gamma$ beam up to a total ionizing dose of $300 \mathrm{kRad}$.

\section{STUDIED COMPONENTS}

We tested wavelength-selective elements in standard planar lightwave circuit (PLC) technology in the silica-onsilicon (SoS) material system and in the experimental silicon-on-insulator (SOI) technology. Both devices are operating in the 1500-1600nm telecom wavelength range. The devices in this experiment were unpackaged. In reality, devices will be packaged of course and a shielding effect may arise.

In interference-based wavelength filters, the passband or stopband centre wavelength, the spacing between the wavelength channels that can be discriminated and the period of the transfer spectrum are set by choosing the basic optical delay length. This delay length is the basic feature of all these filters types whether based on optical feedback (resonators) or not. The filter wavelength (e.g. passband centre) is generally given by

$$
\lambda_{\text {filter }}=\frac{n_{e f f} \Delta L}{m}
$$

where $\Delta L$ is the physical delay length, $n_{e f f}$ is the waveguide modal effective index, and $m$ is the filter order. Here we suppose only one guided waveguide mode is used in the system.

As the optical delay length depends on refractive indices of the waveguide materials, any change in material refractive indices will give rise to a change of the modal effective index and therefore the filter wavelengths will shift. In general, the filter wavelengths considered lie in a relatively small wavelength band (e.g. around $1550 \mathrm{~nm})$. In this wavelength range, $\frac{\Delta L}{m}$ will be of the same order for all considered filter types. Therefore, for a given material system the wavelength shift due to refractive index variations will be approximately the same irrespective of filter design and parameters. Characterization of this shift for one filter design will therefore give a very good idea of the shift in other designs and filter types as well.

As interferometric devices are very sensitive to refractive index variations, even small variations will have a noticeable effect on the transmission spectrum. The refractive index changes introduced by material defects are always paired with material absorption changes, leading to higher insertion loss and different crosstalk behaviour. However, if the changes are very small, the absorption changes will not be noticeable.

In the SoS material system, we tested a 1-by-40 arrayed waveguide grating demultiplexer supplied by OpTun GmbH* Waveguides are formed inside the Silicon dioxide layers by doping the $\mathrm{SiO}_{2}$ with Germanium. The refractive index difference between core and cladding is about $1 \% .^{5}$ By doping with other materials or other techniques, components such as amplifiers can be integrated on the same chip. The component studied here was passive. The channel spacing of the AWG device was 100GHz. The chip was pigtailed to standard single-mode fibers but was not packaged. The die size was about $15 \mathrm{~cm}^{2}$. Fibre to fibre insertion loss is 4 to $5 \mathrm{~dB}$.

A more experimental technology is based on SOI photonic wire waveguides. These are submicron waveguides with a high index contrast, as illustrated in Figure 2(a). Therefore, bend radii of just a few $\mu \mathrm{m}$ can have very low loss resulting to very compact devices. We use $200 \mathrm{~mm}$ SOI wafers with a top Silicon layer of $220 \mathrm{~nm}$ thickness and a $1 \mu \mathrm{m}$ burried oxide. Structures are fabricated using CMOS fabrication methods, including deep UV lithography. ${ }^{3,6}$ The devices tested here are used for studying WDM applications in this technology.

\footnotetext{
*OpTun GmbH, Munich, Germany - www.optun.com
} 


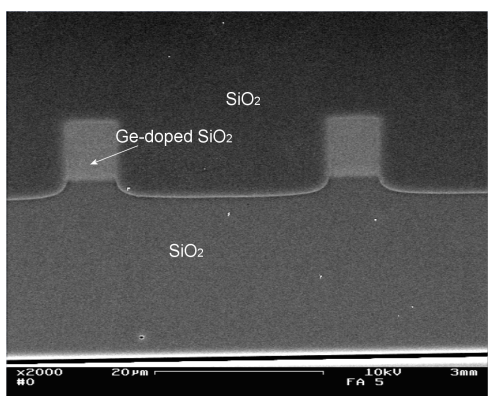

(a) Crosssection of waveguides

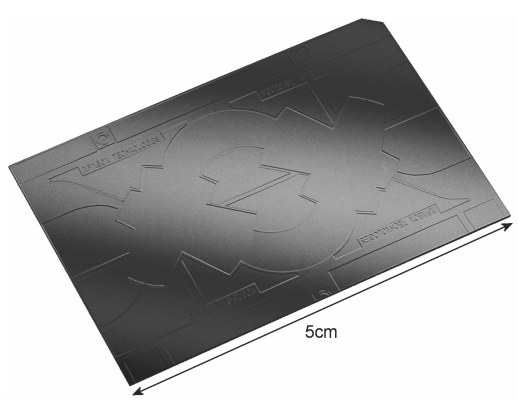

(b) Arrayed waveguide grating

Figure 1. Silica-on-Silicon technology. Pictures (C) 2003 OpTun GmbH

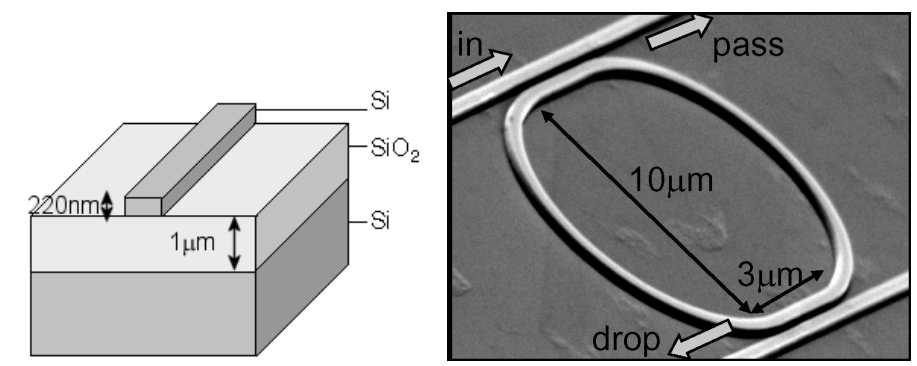

(a) Schematic crosssection of (b) SEM picture of an SOI ring waveguides

resonator

Figure 2. Silicon-on-insulator technology.

The SOI devices characterized were single ring resonators add-drop filters with about $12 \mathrm{~nm}(1.5 \mathrm{THz})$ free spectral range. The device samples were not pigtailed. The die size was about $0.5 \mathrm{~cm}^{2}$, while the component considered was only $0.015 \mathrm{~mm}^{2}$ (including input/output waveguides). Figure 2(b) shows a SEM picture of such a resonator used as an add-drop filter. In a first session, we tested a ring resonator using cleaved waveguide facets for in- and outcoupling (experiment 1, see section 4.2). In a second session, additional ring resonators were measured using vertical fibre couplers (experiments 2 and 3).

\section{MEASUREMENT SETUP}

For optical components, $\gamma$ radiation is generally considered as a worst case with respect to total ionizing dose effects. The facility at ESTEC provides a Co60 $\gamma$ source, radiating photons of 1.173 and $1.332 \mathrm{MeV}$. At the working distance, a nearly collimated beam with a dose rate of about $5 \mathrm{kRad} /$ hour radiates the complete sample, mounted with the component plane perpendicular to the $\gamma$ beam. The dominant ionisation process in irradiated materials is Compton scattering at these energies.

In order to monitor the components during radiation, fibres connect the input and output of the component with a tuneable laser and power meter in the control room. Changes in the coupling efficiency between fibre and sample originating from radiation effects on the fibres can be neglected compared to the effects in the component. A dosimetre was used to monitor the total absorbed dose. As the measured absorbed dose corresponded to the dose in water, we multiplied it by 0.896 to obtain the absorbed dose in Silicon. A $2 \mathrm{~mm}$ thick Aluminum plate prohibits high-energy electrons from impacting the sample and serves as a build up material.

As the SOI components were not fibre-coupled, a fibre alignment setup was used in the facility. For characterisation of the first ring resonator sample, light was coupled in and out cleaved facets through lensed fibres. However, stability of the alignent between fibres and sample was poor and difficult to maintain for the duration of the experiment, therefore insertion losses were increasing during the monitoring period. The facets were coated 


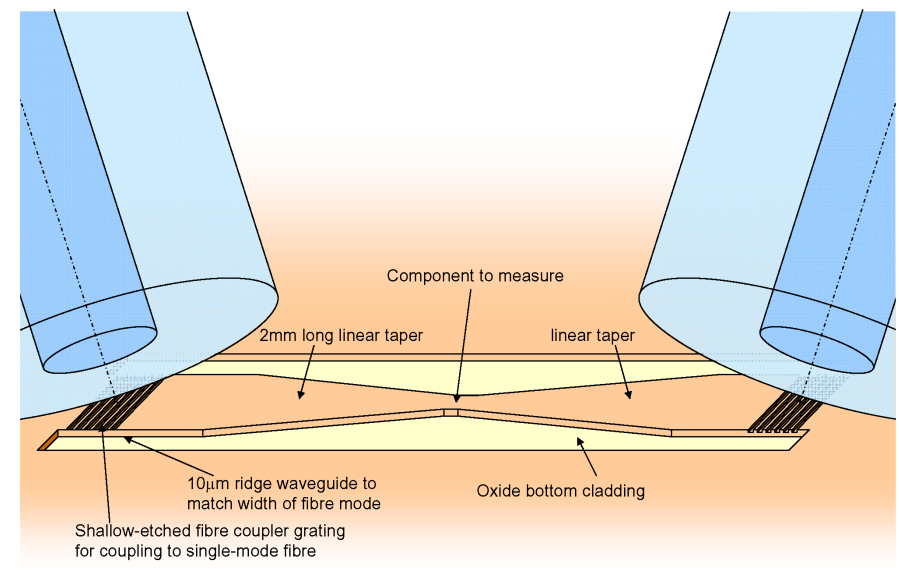

Figure 3. Coupling between single-mode fibre and SOI waveguides using vertical fibre couplers

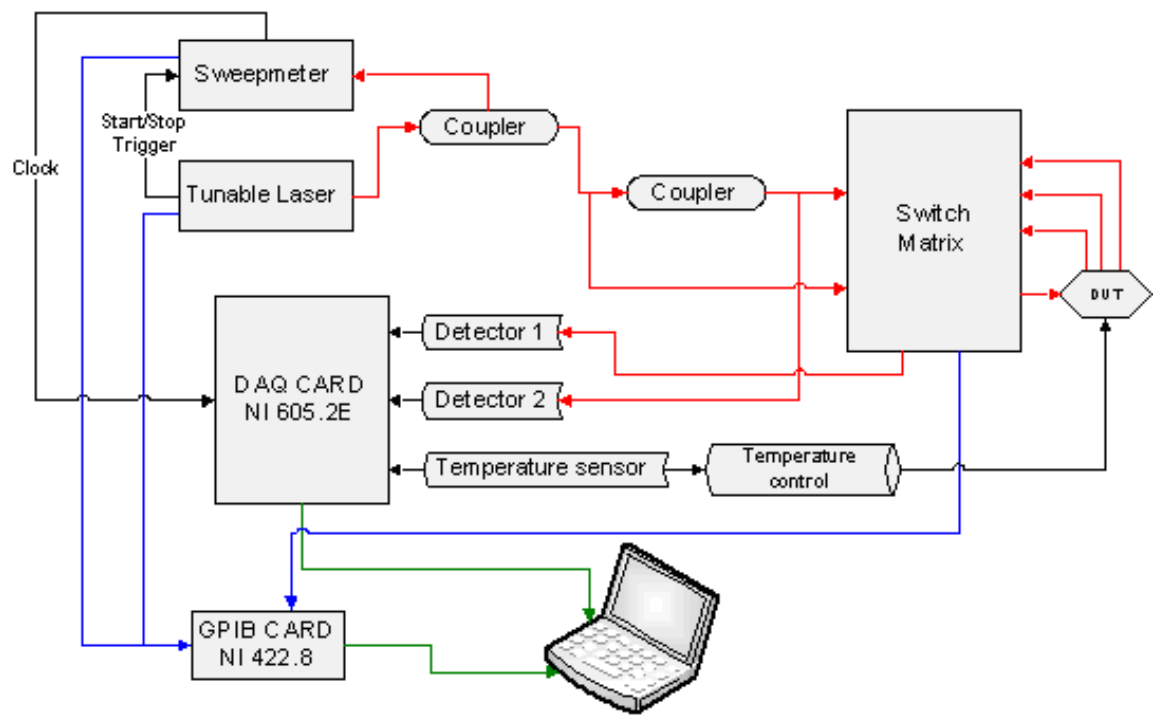

Figure 4. Overview of the measurement setup

with an AR coating. No temperature control of the sample was done, however a record of the room temperature was kept using a thermocouple.

For characterisation of the second series of three ring resonators, vertical fibre couplers were used. ${ }^{7}$ This setup is illustrated in Figure 3. Now light was coupled in and out of the sample with almost vertically mounted standard single-mode fibres. Because these fibre couplers are more tolerant against misalignment, the setup stayed much more stable and monitoring could be done over a longer time period. Temperature control was done using a peltier element and temperature controller in order to keep the components at a constant temperature near room temperature. However, in several of the experiments the temperature controller failed over longer time period for a yet unknown reason.

The measurement setup is illustrated in Figure 4. Only the devices under test (DUT) are exposed to the radiation field. An optical switch matrix is used to be able to measure multiple components sequentially. The switch matrix also allows to measure both in transmission and reflection. To achieve a higher accuracy without increasing measurement speed, a sweep meter is integrated (for high speed wavelength calibration). ${ }^{8}$ The couplers tap laser power towards this sweepmeter and a second detector serving as a reference. The sweep meter 
contains a gas cell providing an accurate optical frequency reference. It uses a Fabry-Perot interferometer to produce periodic frequency intervals. When a sweep is triggered, the tapped tuneable laser input is analyzed by the sweep meter, generating a clock stream that is then used to trigger the data acquisition card. Clock skew and time for acquiring a data sample can be neglected. This setup allows to acquire data with sub pm resolution with a great accuracy. ${ }^{8}$

\section{EXPERIMENTAL RESULTS}

In this paper we present results from two types of devices; an SoS Arrayed Waveguide Grating (AWG) and SOI ring resonators. Each type of device is discussed seperately in the following paragraphs. For both device types, we monitored multiple passband centre wavelengths in transmission during irradiation.

\subsection{Silica-on-Silicon arrayed waveguide grating}

We monitored three outputs of the demultiplexer sequentially by doing three subsequent wavelength scans and changing the optical switch configuration to connect different AWG output fibers to detector 1. As each scan takes a only a few seconds, the scans can be regarded as being done at the same radiation dose. The AWG device was put quite close to the source $(30 \mathrm{~cm})$, resulting in a $157 \mathrm{Rad} / \mathrm{min}$ radiation dose rate in Silicon. To achieve a $300 \mathrm{kRad}$ total dose, a 30 hours experiment time was needed. For each output, we looked at the peak wavelength and the $1 \mathrm{~dB}$ and $3 \mathrm{~dB}$ bandwidths.

Figure 5 shows the transmission spectra of the monitored channels before radiation. In Figure 6 , the peak wavelength difference with respect to the starting value is plotted against the absorbed dose. The first and third channels experienced a shift of $0.03 \mathrm{pm} / \mathrm{kRad}$. For the second channel, a shift of $-0.007 \mathrm{pm} /$ Rad was measured. By monitoring three of the outputs in parallel, we hoped to obtain a reliable result. We do not yet understand why one output port experiences a negative shift while the two others show a positive shift. In any case, the shifts measured are much smaller than typical channel bandwidths even at a total dose of $300 \mathrm{kRad}$. We looked also at the sidelobe levels in the AWG. However, no change was measured, which shows that changes in material absorption are very low in SoS. Therefore, this first experiment indicates that SoS components can be radiationhard and are a very good candidate to be used in interconnect systems. A shielding effect arising from packaging may even improve radiation hardness.



Figure 5. Overlayed transmission spectra of 3 AWG channels

\subsection{Silicon-on-insulator ring resonators}

To characterize the ring resonator devices, we measured the transmission to the drop port. A dose rate in Silicon of $82 \mathrm{Rad} / \mathrm{min}$ was obtained and a 60 hours experiment time was needed to reach a $300 \mathrm{kRad}$ total dose (Experiment 1). During the second session, the setup was changed to put samples a little closer to the source, leading to a 48 hours experiment time with a dose rate of $105 \mathrm{Rad} / \mathrm{min}$ (Experiment 2). Additionally, we now 


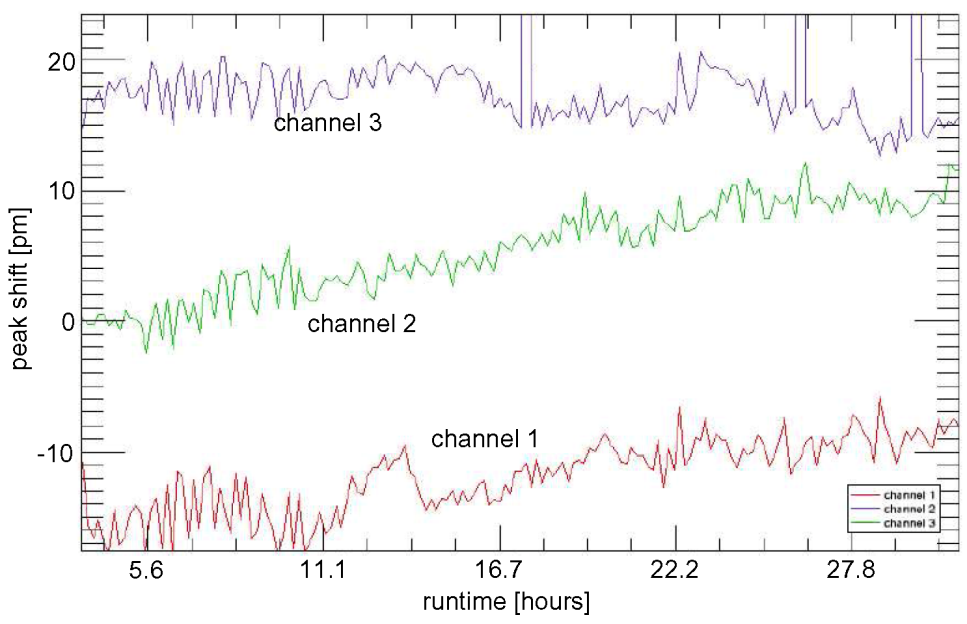

Figure 6. SoS arrayed waveguide grating. Peak wavelength shift of the 3 measured channels as a function of time during irradiation. At the end of the experiment a 300kRad total dose was absorbed.

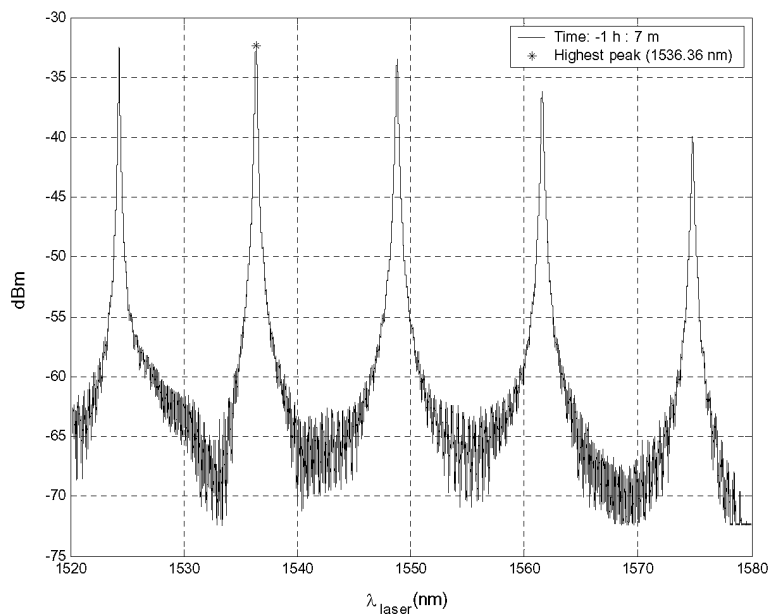

Figure 7. Detected output power spectrum of the drop port of an SOI ring resonator add-drop filter

continued measuring after irradiation was stopped in order to measure relaxation behaviour. Figure 7 shows the output power spectrum from a measured ring resonator device. We monitored the behaviour of several resonance wavelengths in order to get a more reliable result.

To monitor the peak wavelength of a given resonance, a peak detection algorithm was needed. Detecting the $3 \mathrm{~dB}$ transmission wavelengths near that resonance and taking the mean of these proved to be the most accurate and reliable method. An example result of this peak detection is shown in Figure 8.

Figure 9 shows the resonance wavelength shift as a function of absorbed radiation dose and time for the ring resonator add-drop filter used in experiment 1 . The resonator has a $5 \mu \mathrm{m}$ radius and about $12 \mathrm{~nm}$ free spectral range. The $3 \mathrm{~dB}$ resonance bandwidth for this device is about $230 \mathrm{pm}$. No temperature control was done. The result is a quite linear shift for the three resonance wavelengths shown. The ambient temperature is also plotted. We can discriminate the same 24-hour period in both temperature and wavelength shift date. After compensating the wavelength shift data for this temperature effect, we fitted a $0.23 \mathrm{pm} / \mathrm{kRad}$ shift. This is about a factor of 10 higher than the shift in the SoS component.

Figure 10 shows the result from experiment 2, with a ring resonator with the same geometry as the resonator 




Figure 8. Detection of the resonance peak with high-resolution measurements. The changing spectral form of the peak adds some noise to the detected peak trace

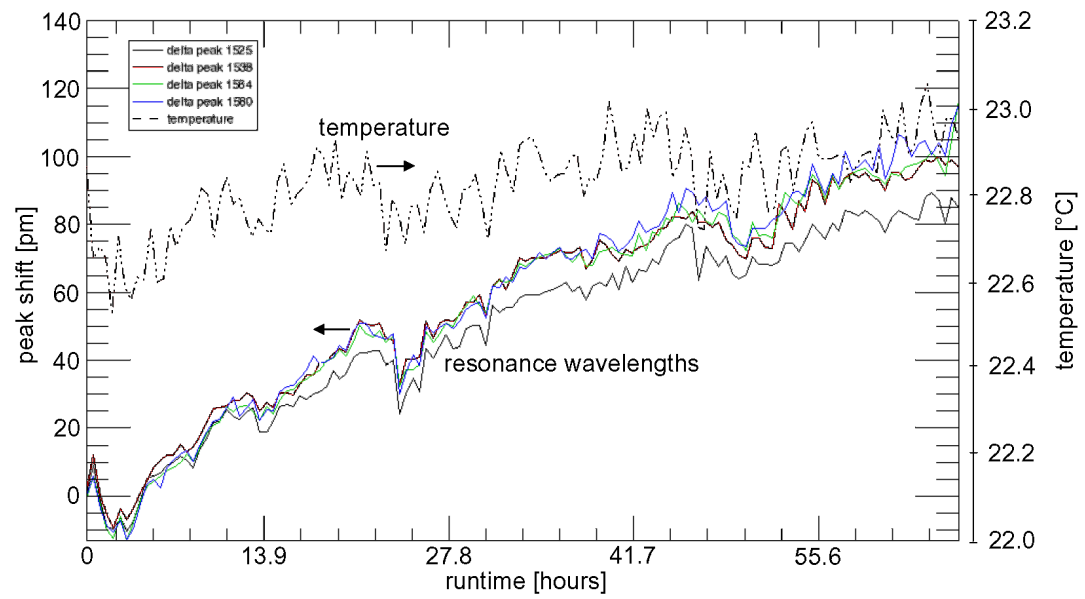

Figure 9. Peak wavelength shift as a function of measured time for an SOI ring resonator, for three resonance wavelengths. The ambient temperature is also plotted. At the end of the measurement, the total obsorbed dose in Si was $300 \mathrm{kRad}$. Experiment \#1 


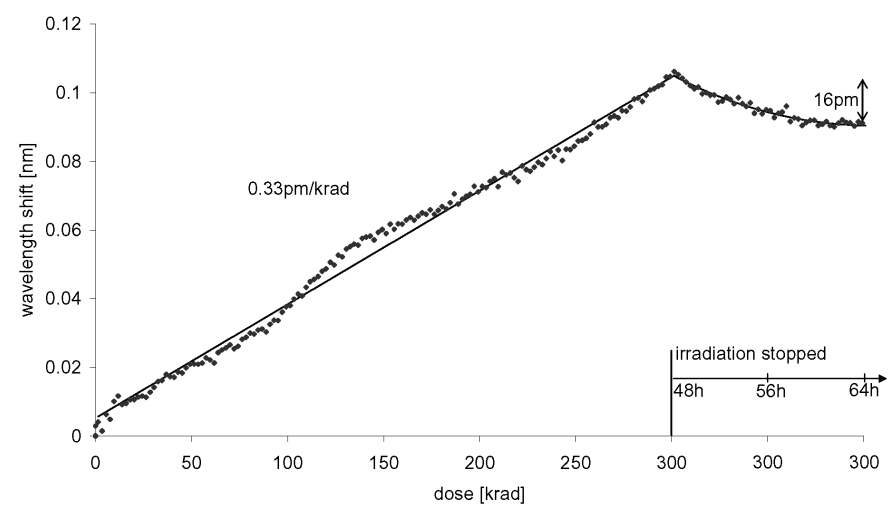

(a) resonance @ 1536nm

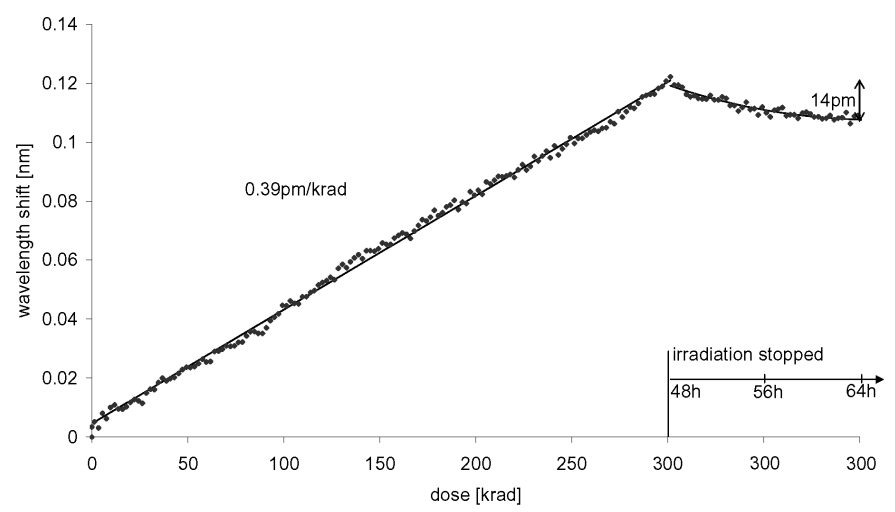

(b) resonance @ $1575 \mathrm{~nm}$

Figure 10. Resonance wavelength shift versus radiation dose for an SOI ring resonator. Experiment \#2

from Figure 9 but with different waveguide widths. Now the sample temperature was kept constant around room temperature. Results for two resonances are shown. We see a very linear resonance wavelength shift during irradiation with a shift of 0.33 and $0.39 \mathrm{pm} / \mathrm{kRad}$. Just a little after irradiation is stopped, the resonance wavelength relaxes somewhat towards the original resonance.

In Figure 11 the result of experiment 3 is plotted, with a third resonator again very much alike the ones used in experiments 1 and 2. However, the temperature control failed after 5.5 hours. This explains the sudden offset of $150 \mathrm{pm}$ (corresponding with a temperature difference of little over $1{ }^{\circ} \mathrm{C}$ ). From then on, we see a drift towards longer wavelengths as in figure 10. However, the curve is fluctuating a lot, making it impossible to get data on the radiation effect. 25 hours after the start of the experiment, the resonance starts drifting to shorter wavelengths. However, this 12-hour period coincides with evening and night time. After 48 hours, irradiation was stopped and a relaxation effect seems to happen like in experiment 2. However, this time period is during evening/night time again, therefore the amount of relaxation is difficult to extract.

The stronger wavelength shift for SOI with respect to SoS was expected due to the larger sensitivity to refractive index changes of the SOI waveguides. However, even in the SOI case the wavelength shift becomes considerable only at very high total doses. We calculated the temperature dependent wavelength shift in these SOI waveguides to be of the order of $100 \mathrm{pm} / \mathrm{K}$. This shows that even at a high total dose, the refractive index change due to radiation can easily be compensated for by slight temperature tuning. As temperature control of passive integrated components is necessary anyway in space, some additional control function would only be needed to enable any radiation induced wavelength drift to be adequately compensated. As the aligment between fibres and chip was slowly degrading over time, insertion loss changes could not be detected. However, additional waveguide 




Figure 11. SOI ring resonator experiment \#3, resonance around $1534 \mathrm{~nm}$. Temperature control failed after 5h30. A temperature dependent drift is superimposed on the radiation effect.

losses due to absorption would also change the extinction ratio between on- and off-resonance wavelengths. Such an effect was not seen during the experiments, which shows that radiation induced losses are neglible. We can conclude that while our experiments show a clear radiation-dependent effect, this is no critical problem for the application of these kind of devices in space.

\section{CONCLUSION}

We measured the $\gamma$ radiation sensitivity of passive waveguides in Silica-on-Silicon and Silicon-on-insulator, two material systems used for photonic integrated circuits. Such integrated components may be a key element in high-speed and high-capacity interconnect applications in space. While commercial Silica-on-Silicon components are available today, high index contrast Silicon-on-insulator components are still experimental. However, the latter can offer more functionality on a smaller die size. The experiments we performed on these devices give an idea on the total ionizing dose effects for a total absorbed dose in Silicon of 300kRad. The main effect of radiation in these materials is a refractive index change. We monitored the passband peak wavelength shift of arrayed waveguide grating and ring resonator components. In Silica-on-Silicon, a wavelength shift of $0.03 \mathrm{pm} / \mathrm{kRad}$ was measured. With respect to typical channel bandwidths, this is an almost neglible change even for high total doses. This indicates that these components can be radiation-hard, for space applications, in the telecom operation wavelength band. The high index contrast Silicon-on-insulator waveguides are more sensitive to refractive index changes, which shows up as a radiation-induced shift that is lower than $0.4 \mathrm{pm} / \mathrm{kRad}$. In this case, a high total dose may lead to an important wavelength shift. However, the temperature dependent shift is very large and can easily be used to compensate for any radiation effects up to very high total dose.

\section{ACKNOWLEDGMENTS}

The authors would like to thank OpTun GmbH for supplying AWG devices.

This work has been carried out in the context of ESA/ESTEC contract 17884/03/NL/HE - Multigigabit Optical Backplane Interconnections. Silicon-on-insulator device study and fabrication has been supported by the EU in the context of the IST-PICMOS project and IST-ePIXnet NoE and by the Belgian IAP-Photon network.

P. Dumon thanks the Institute for the Promotion of Innovation through Science and Technology in Flanders (IWT-Vlaanderen) for a scholarship.

\section{REFERENCES}

1. L. Kazovsky, T. Fong, and T. Hofmeister, "Optical local area network technologies," IEEE Communications Magazine (12), pp. 50-54, 1994. 
2. C. J. Sun, K. M. Schmidt, and W. Lin, "Silica waveguide devices and their applications," in Photonics West 05, (San Jose), January 2005.

3. W. Bogaerts, R. Baets, P. Dumon, V. Wiaux, S. Beckx, D. Taillaert, B. Luyssaert, J. V. Campenhout, P. Bienstman, and D. V. Thourhout, "Nanophotonic waveguides in silicon-on-insulator fabricated with cmos technology," IEEE Journal of Lightwave Technology 23, pp. 401-412, January 2005.

4. M. Naydenkov and B. Jalali, "Advances in silicon-on-insulator photonic integrated circuit (soipic) technology," in IEEE International SOI Conference, p. 56, October 1999.

5. "Optun integrated optics - white paper," March 2003. http://www.optun.com/archive/white_paper.pdf.

6. P. Dumon et al., "Low-loss photonic wires and ring resonators fabricated with deep uv lithography," IEEE Photonic Technology Letters 16, pp. 1328-1330, May 2004.

7. D. Taillaert, W. Bogaerts, P. Bienstman, T. Krauss, P. V. Daele, I. Moerman, S. Verstuyft, K. D. Mesel, and R. Baets, "An out-of-plane grating coupler for efficient butt-coupling between compact planar waveguides and single-mode fibers," IEEE Journal of Quantum Electronics 38(7), pp. 949-955, 2002.

8. J. Ensher and C. Myatt, "Fast, accurate wavelength calibration for optical component testing." PXI Technology Review, 2002. 